\title{
Setecientosmonos y la modernización de la crítica literaria argentina
}

\author{
Setecientosmonos and the Modernization of Argentinian Literary Criticism
}

Setecientosmonos (setecentos macacos) e a modernização da crítica literária argentina

\section{Judith Podlubne}

UNIVERSIDAD NACIONAL DE ROSARIO; CONICET, ARGENTINA

Profesora de la Universidad Nacional de Rosario e Investigadora en

CONICET, Argentina. Doctora en Letras, Universidad de Buenos

Aires. Autora de Escritores de Sur. Los inicios literarios de fosé Bianco

y Silvina Ocampo (Rosario: Beatriz Viterbo Editora/UNR, 2011), y

editora junto con Martín Prieto de María Teresa Gramuglio, la exigencia

crítica: quince ensayos y una entrevista (Rosario: Beatriz Viterbo Editora/

UNR, 2014). Ha publicado numerosos artículos en varias revistas

especializadas. Correo electrónico:judithpodlubne@gmail.com

Artículo de reflexión

El artículo recoge las conclusiones parciales obtenidas, por un lado, en el marco de la investigación individual que realizo en CONICET (2014-2015), titulada "Renovación intelectual, actualización teórica y 'resistencia a la teoría' en el período de modernización de la crítica literaria en Argentina", y, por otro, en el marco del Proyecto de Investigación Plurianual (PIP 2013-2015) titulado: "La resistencia a la teoría en la crítica literaria en Argentina: algunos episodios desde 1960 hasta la actualidad" (Director: Dr. Miguel Dalmaroni. Co-directora: Judith Podlubne. Investigadores integrantes: Dr. Alberto Giordano, Dra. Analía Gerbaudo y Dra. Julieta Yelin).

Documento accesible en línea desde la siguiente dirección: http://revistas.javeriana.edu.co

doi: $10.11144 /$ Javeriana.cl20-39.smcl 


\section{Resumen}

El artículo presenta una lectura crítica de la revista Setecientosmonos, publicada en la ciudad de Rosario (Argentina) entre 1964-1967, y aún poco explorada por los lectores especializados. La hipótesis que orienta esta lectura afirma que el ingreso de Nicolás Rosa, a fines de 1964, refunda la publicación. Rosa convierte una revista de jóvenes inexpertos, sin contactos con el mundo literario, en otra, también de jóvenes, ansiosos por asumir posiciones intelectuales y acercamientos teórico-críticos a la literatura. Bajo su influencia, Setecientosmonos adquiere el carácter diferencial que la vuelve un episodio significativo del proceso de modernización teórica de la crítica literaria argentina.

Palabras clave: Setecientosmonos; literatura; política; modernización teórica; crítica literaria

\section{Abstract}

This article presents a critical reading of the magazine Setescientosmonos, published in Rosario (Argentina) between 1964-1967, and which has not been thoroughly explored by specialized readers. The hypothesis which guides this reading states that the inclusion of Nicolás Rosa at the end of 1964 re-founded the publication. Rosa transformed a magazine of inexperienced youths, without contacts in the literary world, into another, also belonging to youths eager to assume intellectual positions and theoretical approaches to literature. Under his influence Setescientosmonos acquires the differential character that turned it into a significant episode of the theoretical modernization process of Argentinian literary criticism.

Keywords: Setecientosmonos; literature; politics; theoretical modernization; literary criticism

\section{Resumo}

$\mathrm{O}$ artigo apresenta leitura crítica da revista Setecientosmonos, publicada na cidade de Rosario (Argentina) entre 1964-1967, e ainda nem tão explorada pelos leitores especializados. A hipótese que orienta essa leitura afirma que o ingresso de Nicolás Rosa, a finais de 1964, refunda a publicação. Rosa converte uma revista de jovens inexperientes, sem contatos com o mundo literário, em outra, mesmo de jovens, ansiosos por assumir posições intelectuais e aproximações teóricocríticas da literatura. Sob a sua influência, Setecientosmonos adquire carácter diferencial que a torna episódio significativo do processo de modernização teórica da crítica literária argentina.

\section{Palavras-chave:}

Setecientosmonos; literatura; política; modernização teórica; crítica literária

\section{Cómo citar este artículo:}

Podlubne, Judith. "Setecientosmonos y la modernización de la crítica literaria argentina". Cuadernos de Literatura 20.39 (2016): 270-

295. http://dx.doi.org/10.11144/Javeriana.cl20-39.smcl 
A Comienzos De los años 9o, durante una conocida mesa redonda dedicada a debatir el rol de las revistas culturales en la primera década democrática, Nicolás Rosa decía: "Creo que las revistas - sobre todo las revistas literarias, aunque todas las revistas culturales terminan siendo una revista literaria- son prácticamente la autobiografía de la literatura, la autobiografía de la historia de la literatura. [...] A veces una autobiografía un tanto novelada y simultáneamente muy íntima, muy personal e incluso algo chismosa" ("El rol de las revistas culturales" vii). Enunciada con cariz tentativo y sin escatimar la provocación modernista ("todas las revistas culturales terminan siendo revistas literarias"), la ocurrencia, que sin dudas le debía mucho a las tesis de El arte del olvido, publicado dos años antes, anunciaba la posibilidad de un trato diferente para las publicaciones periódicas. El punto de vista autobiográfico proponía que las revistas no solo se leyesen como medios institucionales de la actividad cultural de una época sino también como el relato vivencial de los intercambios intelectuales y afectivos entre integrantes y colaboradores. Al interés por sus políticas editoriales, sus posiciones de grupo, sus estrategias de inserción en el campo intelectual, su recorte de público, se sumaba la curiosidad por el registro de contingencias, recelos, acuerdos y disputas, que manifiestan los textos, sobre todo los textos colectivos, cuando se los interpela con ánimo archivístico.

El acto de archivar, enseña Derrida (24), crea tanto como registra el acontecimiento. El archivo no es el lugar de almacenamiento de un contenido que existiría de todos modos, tal y según se cree que fue. Como el "acto autobiográfico" (Rosa, El arte del olvido 51), el de archivar no se agota en el rescate y la preservación de un sentido realizado en el pasado, sino que experimenta el carácter estructuralmente inconcluso de ese pasado, lo abre a las transformaciones futuras y permite que lo sucedido retorne como tal vez nunca ocurrió. Archivo y autobiografía comprometen una temporalidad similar, la de lo todavía porvenir del tiempo sido, para la cual el sentido que vuelve, lo que se recuerda o reconstruye, necesita de la imaginación presente para poder instituirse. Impulsada por estas especulaciones, la ocurrencia de Rosa interrogaba sin proponérselo la sentencia que Beatriz Sarlo establecía durante el mismo debate. "No hay nada más viejo que una revista vieja", afirmaba, con la razón parcial que le confería remitir estas publicaciones a la actualidad:

$[\ldots]$ los que hacemos revistas [y] los que estudiamos revistas como parte de las textualidades que se estudian en historia literaria, en historia de las ideas, en historia intelectual, sabemos que una revista vieja es algo amarillento, no sólo desde el punto de vista de sus colores, [sino] porque todo aquello que fue nuevo, que fue experimental, que pasó por el banco de prueba, por el laboratorio y se sostuvo lo encontramos en los libros, pasó a los libros. Y 
aquello que no se sostuvo da ese color amarillento que tiene la revista vieja. ("El rol de las revistas culturales" xii)

Su experiencia al frente de revistas importantes (Los libros y Punto de vista) y su amplia competencia en investigaciones sobre publicaciones periódicas la autorizaban a juicios concluyentes y controvertidos (entre ellos, considerar al libro como prueba de un sentido probado y perdurable) que su exposición desarrollaba apelando al Benjamin de la era de la reproductibilidad técnica:

Es como si en la revista vieja se produjera la pérdida del aura, la revista tiene su aura en el presente, no está tensionada hacia el futuro... Los textos pueden estar tensionados hacia el futuro, pueden tener ambición de futuro y ambición de permanencia en un tiempo disparado hacia adelante, pero la revista logra su aura en el presente. Transcurrido el presente el aura se desvanece. ("El rol de las revistas culturales" xii)

La asimilación del presente a lo actual, promovida por la del aura a lo nuevo, clausuraba la posibilidad de que las revistas se leyeran como algo más que documentos de cultura, al tiempo que olvidaba las lecciones de Benjamin en sus fragmentos sobre el concepto de historia. La idea benjaminiana de que la verdadera imagen del pasado adviene bajo la forma de la rememoración, y no por obra de la memoria, una idea cuya matriz evidente es Marcel Proust, impugna las convicciones historiográficas para reemplazarlas por la temporalidad del archivo y la autobiografía.

Mientras las conclusiones de Sarlo cancelaban los posibles del pasado al restringirles ambición de futuro, el enunciado de Rosa los reinstalaba en el presente como virtualidades por definirse. Su ocurrencia daba lugar a una convicción: las revistas no solo testimonian un tiempo anterior sino que además lo rememoran para el lector del presente que, interpelado por la indefinición, se resiste a derogarla. La perspectiva autobiográfica compone el relato vivencial, disperso en estas publicaciones, atraída por el rumor sedicioso, aún activo, que agita el sentido de las anécdotas e impresiones que lo integran. Como si aspirara a dar con el "aura autobiográfica" de estos textos, a encontrarse, diría Benjamin, con "la imagen en que lo sido comparece con el ahora" (121), la lectura procura devolver a las revistas culturales el carácter poroso e irresuelto que la mirada historicista suele cristalizar (o deformar) en certezas. Es probable que a esa aspiración responda la circunstancia de que Rosa dedicara un segmento significativo de su intervención en el debate a figurar su mito de origen como crítico en una olvidada revista de provincia, editada a mediados de los años 60. 
Nacida del ímpetu juvenilista de un grupo de amigos aficionados a la literatura, Juan Carlos Martini, Carlos Schork, Omar Pérez Cantón y Rubén Radeff, Setecientosmonos toma su nombre de un chiste cándido y zumbón, que convierte en blanco de burla la figura fácil del profesor conferencista, mientras anticipa sin saberlo el tono principal de los números iniciales. ${ }^{1}$ Con la misma miopía que parodian, y tan proclives al malentendido como el profesor de la anécdota, los miembros de la revista se ríen de una torpeza y una solemnidad similares a las que transmiten los cuentos y poemas primerizos que escriben y publican en esos números. Setecientosmonos surge del ánimo vocacional de cuatro principiantes, amigos del barrio y compañeros de facultad. ${ }^{2}$ Según se desprende de sus relatos, Martini y Schork comienzan a sentirse escritores solo después de haber ganado el concurso de cuentos que, a mediados de 1964, organiza Amigos del Arte (sede Rosario) con el auspicio del Fondo Nacional de las Artes. Para ese momento la revista cuenta ya con dos números. Además de un rito de iniciación literaria, este concurso les depara el encuentro con quien en adelante marca el rumbo editorial de la publicación. En la entrega de premios conocen a Nicolás Rosa, por entonces un joven estudiante del profesorado de Letras en la Universidad Nacional del Litoral (sede Rosario), ya muy vinculado al medio cultural de la ciudad. La incorporación de

1 El chiste, un lugar común en la Argentina entre las décadas del 60 y 70, encabeza el primer volumen y dice lo siguiente: "Era un profesor viejo y miope que trasuntaba en su aspecto mientras dictaba la conferencia, el nerviosismo de saber lo profundo y escabroso del tema. Los alumnos, sentados, escuchaban en un ambiente tenso, hasta que se pararon para oír con admiración los revolucionarios conceptos de la exposición que ya concluía:

-... las masas llegaran a su cometido. La conferencia ha terminado. Setecientosmonos.

El alumnado salió de su silencio, para elevar un murmullo de incomprensión. El profesor se movió inquieto y acercó un poco más el papel a sus ojos.

- Setentamonos - leyó. Y el murmullo volvió a alzarse.

El papel estuvo muy cerca de su nariz cuando volvió a leer:

- Sietemonos.

Posiblemente los comentarios fueron indignados o desaprobatorios, porque entonces el profesor se calzó los anteojos y sonrojándose leyó:

- Sentémonos.

2 Martini, Schork y Radeff crecen en la misma manzana del barrio Pichincha de la ciudad de Rosario (Argentina), en la calle Ricchieri entre Córdoba y Rioja. Martini y Schork empiezan juntos a estudiar Derecho; conocen a Pérez Cantón en la facultad, en el edificio de Bvd. Oroño y 3 de febrero, que para ese momento Derecho comparte con Ciencias Económicas. Radeff es hijo del dueño de la librería La Médica, donde se imprimen los apuntes de ambas carreras. Cfr. "Entrevista a Rubén Radeff" de Julieta Tonello, publicada en http://antologiasetecientosmonos.blogspot.com.ar/2012/12/entrevista-ruben-radeff.html, "Liminar" de Juan Martini y "Entrevista a Carlos Schork" de Osvaldo Aguirre y Julieta Tonello, incluidos en Setecientosmonos. Antología. Debo a la publicación de esta antología y a las conversaciones con sus editores el interés renovado por la revista. 
Rosa se produce en los últimos meses de ese año y transforma de manera sustancial el tenor de la revista. Varias décadas más tarde, Martini y Schork todavía celebran la incidencia renovadora que aquel encuentro tuvo sobre sus formaciones literarias e ideológicas. Pérez Cantón, en cambio, recuerda el malestar que le provocaron las iniciativas de Rosa y atribuye a esa incomodidad las razones de su alejamiento. "El ingreso de Nicolás Rosa, [cuenta Martini (7)] cambió todo. Desde el logotipo [...] (hasta hoy, agrega, a mí me sigue gustando el dibujo gótico que diseñó Omar Pérez) hasta los colaboradores habituales. Nicolás, en rigor, dirigió orgánicamente Setecientosmonos desde el primer momento de su llegada".

Sin embargo, la incorporación de Rosa al staff de directores se concreta recién en el número 6 (agosto 1965); antes fue "colaborador permanente", en el número 3/4 (sept./dic. 1964), y "secretario de redacción", en el número 5 (abril 1965). Bajo su influencia, la revista adquiere el carácter diferencial que la vuelve un episodio significativo del proceso de modernización teórica de la crítica literaria argentina. Los primeros dos números tienen una realización artesanal, rudimentaria, con tapas básicas (la del número 2, julio 1964, algo mejorada en relación con la primera, incorpora fotos y color), algunas erratas y unas pocas ilustraciones elementales a cargo de Pérez Cantón. Se tipean en una máquina de escribir prestada, en las oficinas de la sección Alumnado de la Facultad de Derecho, y se imprimen en el mimeógrafo de la librería La Médica. Los directores se ocupan de todo: mecanografían, diagraman, dibujan, pican los esténciles, tiran las copias. El número siguiente registra el paso a la imprenta. El padre de Radeff, que había comenzado a publicar libros de medicina, los pone en contacto con el dueño de la imprenta Molachino, quien les financia la edición del número 3/4 hasta tanto consigan avisos publicitarios y vendan los ejemplares. El rediseño gráfico es evidente. Por primera vez, el sumario aparece en tapa, se inicia la diagramación de páginas en columnas, se incorpora variedad de tipografías y se incluyen fotos e ilustraciones firmadas por artistas locales, Mele Bruniard y Oscar Herrero Miranda. A partir del número 6, las fotos reemplazan casi por completo las ilustraciones. La edición de imprenta anuncia los cambios que la revista desarrolla en adelante.

Rosa refunda Setecientosmonos. Convierte una revista de jóvenes entusiastas e inexpertos, casi sin contactos con el mundo literario, en otra, también de jóvenes, ansiosos por asumir posiciones intelectuales y acercamientos teóricocríticos a la literatura. La memoria de los protagonistas recupera con indulgencia los números previos a su ingreso. Aunque de escaso valor literario o cultural, esos números funcionarían como umbral necesario de las transformaciones que luego experimenta la revista. "Con el tiempo pensé [escribe Martini (7)], que 
me hubiera gustado que Setecientosmonos hubiera empezado en el nro. 3/4. Más adelante pude aceptar que sin esos dos primeros números casi irresponsables [...] no hubiese pasado lo que pasó después con la revista". Lo cierto es que, más allá de la condescendencia que el recuerdo de Martini comparte con el de Perez Cantón, para quien "lo posterior [tampoco] hubiese sido posible sin esa etapa de estudiantina" (Perez Cantón 347), ni las cartas editoriales ni las colaboraciones publicadas hacían preveer el rumbo que la revista manifestó a partir del nro.3/4 y asumió decidida a partir del 6 .

Los diez números de la colección se publican entre mayo de 1964 y octubre de 1967. Los primeros cinco aparecen encabezados por "Cartas de la dirección" que ofician de notas editoriales y enuncian los enfoques compartidos. La carta del número $3 / 4$ exhibe una conciencia plena de los déficits anteriores. Los primeros párrafos evalúan las carencias iniciales en términos compensatorios, similares a los que Martini y Pérez Cantón suscriben todavía, y justifican la salida de un primer número "poco representativo de [sus] aspiraciones y falto de calidad en algunos casos", amparados en la doble consigna de que "las revistas se hacen sobre la marcha" (nro. 3/4, 1) y la marcha, previsiblemente, es progresiva y superadora. Los párrafos siguientes anuncian una nueva etapa cuyos propósitos no se terminan de formular con claridad. La periodización en etapas atiende a las circunstancias compartidas por los integrantes, pero resulta poco convincente para evaluar el curso de la revista. Con el ingreso de Rosa, Setecientosmonos no refuerza un perfil previo sino que proyecta uno nuevo. Los números 3/4 y 5 presentan cambios significativos que advierten sobre el camino elegido: un evidente retroceso del impresionismo crítico, representado por Carmelina Rivero de Castellanos, un personaje de cierto renombre en el ambiente literario local, la incorporación de escritores de relevancia nacional y un repentino interés por la política ${ }^{3}$.

Durante los números iniciales y hasta el 3/4, en el que pasó a ser colaboradora permanente, Carmelina Rivero de Castellanos integró el staff de Setecientosmonos, en calidad de "aserora literaria", y tuvo a su cargo la sección "Bibliográficas". El número 5 todavía la registra entre los miembros pero ya no incluye participaciones suyas; de allí en adelante no volverá a aparecer en la revista. La sección "Bibliográficas", que a partir del nro. 6 asume el nombre algo más específico de "Crítica", la firman Rosa, Alberto Laguna y Martini en esa

3 Carmelina Rivero de Castellanos fue profesora de Latín en la Facultad de Filosofía y Letras de la Universidad del Litoral, a comienzo de los años 50; colaboradora literaria del diario $\mathrm{El} \mathrm{Li}$ toral de Santa Fe y ganadora del Premio Municipal de Literatura de Rosario "Legado Manuel Musto" en 1956, con el libro de cuentos La puerta colorada. 
oportunidad. Como señala Osvaldo Aguirre (12), en el comienzo Carmelina de Castellanos fue la única redactora con antecedentes literarios, orientó las primeras lecturas y vinculó a los integrantes de la revista con escritores conocidos: Ernesto Sábato y, especialmente, Abelardo Castillo:

A través de Carmelina [recuerda Schork (338)] tuvimos conocimiento de $\mathrm{El}$ escarabajo de oro, revista emblemática de aquel entonces. Con Juan Carlos empezamos a viajar a Buenos Aires y a asistir a las reuniones que se hacían en el Tortoni alrededor de Abelardo Castillo. Así conocimos a Liliana Heker, Ricardo Piglia, Vicente Battista, Miguel Briante y a tantos otros... que nos aportaron experiencia y nuevas ideas.

Las reuniones del Tortoni inspiraron las del bar en el Hotel Savoy de Rosario, donde los monos (así se autodesignaron) se juntaban semanalmente ${ }^{4}$. Quizás la rúbrica zoológica en el nombre de la revista y el subtítulo ("revista literaria, con anteojos") también deba alguna inspiración a las publicaciones de Castillo ${ }^{5}$.

Los escritores de El escarabajo de oro tuvieron una influencia inicial y relativa en Setecientosmonos. A los vínculos de Carmelina de Castellanos, debieron sumarse los del escritor Alberto Lagunas, corresponsal de El escarabajo... desde el primer número (junio 1961), miembro del Consejo de redacción a partir del 28 (abril 1965) y colaborador de Setecientosmonos desde el segundo ${ }^{6}$. Castillo publicó dos cuentos en la revista: "Conejo", que ya había aparecido en Las otras puertas (Buenos Aires: Goyanarte, 1961), en el primer número, y "Requiem para Marcial Palma", recogido al año siguiente en Cuentos crueles (Editorial Jorge Álvarez, 1966), en el número 7. Como él, también participaron otros integrantes de El escarabajo...: Arnoldo Liberman, Humberto Constantini, Marta Lynch, Fernando Quiñones y Marta Goldín. El número doble incluyó una entrevista a Castillo y la mención elogiosa de su participación en un malogrado ciclo de charlas y debates organizado en Rosario por los directores de Setecientosmonos. La entrevista reúne los motivos habituales del credo intelectual del escritor: la importancia de la profesionalización, el compromiso, la eficacia de las revistas literarias, Sábato, la prosa de Borges, Cortázar. La solemnidad de Castillo y alguno

4 A partir del nro. 2 y hasta el sexto incluido, la revista agregó la siguiente leyenda: "se reciben colaboraciones en richieri 888, o los viernes desde las 22: del savoy los viernes desde las $22 \mathrm{hs} /$ nos sufre y responde por nosotros: mozo don pedro sosa"

5 El escarabajo de oro agregaba a su nombre la cláusula "revista sospechosa". Para una caracterización de la revista, ver Calabrese.

$6 \mathrm{Al}$ mes siguiente de ingresar al Consejo de Redacción de El escarabajo de oro, Lagunas se incorpora al staff de Setecientosmonos como "colaborador inmediato" en el nro. 5. 
de esos y otros motivos reconocibles, entre los que se cuentan sus disquisiciones partidarias, su adhesión a Sartre, son parodiados pocas páginas después en la entrada al humorístico "Diccionario biográfico de la literatura argentina"7 . El cruce de reconocimiento y parodia cifra en un mismo número el vínculo de relativa proximidad que los monos entablan con el escritor.

\section{Literatura y política}

Excluido de los dos primeros números, el interés por la política señala el cambio más significativo que se produce en ese momento. Las "Cartas de la dirección" con que se abren los números iniciales se desentienden explícitamente del asunto y anuncian una convocatoria sin restricciones: "Nos mueve una inquietud desprovista de intereses políticos o lucrativos, y anhelamos nuclear en forma amplia a todos aquellos que en mayor o en menor medida sientan vocación e interés por la literatura y sus expresiones" (nro. 1, s/n). En el número 2, aparece además un artículo de José María Gantus, "Mensaje y compromiso", que con didactismo ingenuo discute la noción sartreana, apelando a la fórmula de "literatura responsable", acuñada por Guillermo de Torre a fines de la década del 40 con la pretensión de ajustar el concepto de Sartre. Como señaló Osvaldo Aguirre (16), la inquietud política ingresa a Setecientosmonos con la primera colaboración de Rosa, un artículo sobre Cabecita negra, de Germán Rozenmacher, publicado en el nro. 3/4, y se ratifica con la publicación del suplemento Testimonios (mayo 1965), dedicado a rechazar la invasión estadounidense a República Dominicana ${ }^{8}$. El artículo de Rosa obedece a la reedición de Cabecita negra aparecida en Jorge Álvarez en 1963. Ganador del concurso de cuentos de El escarabajo de oro el año anterior, el libro recibe una primera edición en un sello pequeño y desconocido. ${ }^{9}$ Castillo promueve la narrativa de Rozenmacher en la entrevista

7 El "Diccionario..." simula ser la transcripción de un documento inédito, manuscrito y de autor desconocido, encontrado por los redactores de la revista "en su búsqueda incesante por el Archivo General de la Nación, la Biblioteca Nacional y el Archivo de Armas". Recoge las entradas correspondientes a las primeras letras del abecedario y, además de la dedicada a Castillo, se incluyen otras a Bioy Casares y Borges, entre otros.

8 Aunque se anuncia como el primero de una serie destinada a "asumir nuestra responsabilidad con el momento y con los acontecimientos actuales", el suplemento Testimonios se publica por única vez. Rosa figura entre los directores, junto a Martini y Schork, lo que permite conjeturar que se trata de una idea suya, y escribe una nota de opinión política titulada "Una agonía histórica", en la que denuncia los abusos del imperialismo inglés y norteamericano. En su formato y objetivos, el suplemento recuerda los Cuadernos editados por la revista Contorno.

9 Se trata de Ediciones Anuario. La tirada de 1500 ejemplares se agota de inmediato. $\mathrm{Al}$ año siguiente, la editorial Jorge Álvarez abre sus puertas con la reedición. "Así arranqué [recuerda Álvarez (36)]. Lo primero que hice fue precipitarme tras un libro de Germán Rozenmacher, 
de Setecientosmonos cuando le preguntan sobre los cuentistas del momento. Los argumentos de Rosa se inscriben en el marco de la revisión del peronismo, abierta con el derrocamiento en 1955 y alcanzan un doble carácter inaugural: instala la política como preocupación persistente en la revista, a la vez que manifiesta reservas y objeciones hacia sus capacidades analíticas y explicativas. "Las actuales generaciones argentinas [afirma Rosa] viven un momento de reflexión. Fenómeno tan importante como la época peronista es una suerte de barrera que impide toda conexión con el pasado. Se trata, ahora, de comprender" (nro. 3/4, $3)^{10}$. La voluntad comprensiva, herencia de las lecturas contornistas, vigente en amplios sectores de la izquierda, domina la primera mitad de la nota. Rosa revisa el sentido de la época desde la convicción de que la literatura habría conseguido lo que ni la política ni la sociología ni la economía pudieron:

El análisis del fenómeno peronista ha sido realizado por políticos, la mayor parte de las veces sin ninguna lucidez y siempre muy interesadamente, explicado por sociólogos y valorado por economistas. La literatura, la preocupada literatura argentina contemporánea, se ha abocado necesariamente a explicarse a sí misma a través de su pasado inmediato. Era la única salida. (3)

El enunciado final introduce un punto de fuga sutil a sus argumentos. En la idea de que la literatura se explica a sí misma a través de la revisión de su pasado y en ese movimiento se conecta con la historia (con la complejidad del fenómeno peronista, en este caso), reverbera la tesis central del Roland Barthes de Le degré zéro de l'écriture. La propuesta barthesiana de que el autocuestionamiento de la literatura, la interrogación sobre su propio estatuto institucional, es un índice histórico de conflictos políticos, resultará una divisa para el Rosa que, en 1967, traduce el libro de Barthes para la editorial Jorge Álvarez.

Sin embargo, a pesar de esta resonancia suelta, que alerta sobre las miras del pensamiento crítico de Rosa, sobre su precoz interés en la especificidad política de lo literario, sus argumentos manifiestan todavía una enunciación convencional, apegada a valoraciones envejecidas.

Nuestra literatura actual ha puesto en vigencia la problematización del fenómeno con más energía que ninguna otra fuerza del país. La política escamotea la realidad; la economía la circunscribe; la sociología termina por

Cabecita negra, toda una verdadera apuesta medio transgresora. Incluso si se piensa en relación con el título era una apuesta ideológica...". Sobre la narrativa de Rozenmacher, ver Olguín y Zeiger.

10 En adelante y hasta tanto se señale lo contrario, las citas pertenecen a este artículo, por tanto solo se consigna el número de página entre paréntesis. 
esquematizarla. La literatura, por lo menos ese ha sido el intento de algunos, ha querido ir a las causas más profundas, a la motivación interna del suceso para explicarlo como lo que en realidad es: una realidad humana total (4).

Asociada a la idea de literatura como vía de conocimiento, como modo de acceso a causas fundamentales, sus juicios defienden el carácter explicativo de las producciones literarias. Última expresión de una serie integrada por Ernesto Sábato, David Viñas, Manuel Gálvez, Beatriz Guido, Elvira Orphée y algunos otros, Rozenmacher exploraría el problema del peronismo desde un ángulo novedoso. El valor del relato - Rosa solo se detiene en "Cabecita negra" atraído por la "urgente temática" que desarrolla y no analiza los otros cuentos- residiría en componer con "dolorosa objetividad narrativa" la psicología de un personaje representativo del sector social que particulariza las masas peronistas y las distingue de otras experiencias europeas o latinoamericanas similares. "Cabecita negra" permitiría conocer todo un "dramático esquema social a través de un hecho particular y único". "Poder concretar estéticamente fenómeno tan complejo [concluye Rosa] prueba una vez más que la literatura social no implica negar necesariamente los valores estéticos"(6).

La conclusión, elocuente en varios sentidos, manifiesta, por un lado, el cruce sobre el que se constituye la figura del crítico literario en su primera etapa y anuncia, por otro, la inflexión que sus intervenciones le imprimirán al vínculo entre literatura y política en la revista. Así como la apelación a valores estéticos resulta, a mediados de la década del 6o, el síntoma activo de una formación tradicional, la disposición a apreciar los problemas formales específicos, en lo que su tradicionalismo sigue designando como "literatura social", subraya el apego de Rosa al modo de la crítica literaria inaugurado por los denuncialistas a comienzo de la década anterior y desarrollado en el Instituto de Letras de la Universidad Nacional del Litoral (sede Rosario), durante la dirección de Adolfo Prieto ${ }^{11}$. Los vínculos de Rosa con el

11 Prieto dirigió el Instituto de Letras de la entonces llamada Facultad de Filosofía, Letras y Ciencias del Hombre de la Universidad Nacional del Litoral desde 1959 y hasta 1966, cuando renunció tras la intervención de la dictadura de Onganía a las universidades nacionales. La sede de la universidad funcionaba en la ciudad de Santa Fe pero la carrera de Letras, junto con otras, se dictaba en Rosario. La Universidad Nacional de Rosario se creó poco después, en 1968. Además de ser director del Instituto, Prieto fue decano de la facultad y profesor titular de la cátedra de "Literatura Argentina". La relación de continuidad y desarrollo que él y otros docentes del Instituto, entre los que se contaba David Viñas, establecieron con los modos de leer inaugurados por Contorno resultó decisiva para las transformaciones que se produjeron en la carrera. Viñas comenzó a dictar clases en Rosario en 1957, cuando se le designó profesor interino de "Introducción a la Literatura" y de "Literatura Argentina" (la cátedra que desde 
Instituto de Prieto, su participación en actividades organizadas en ese ámbito, son anteriores al ingreso en Setecientosmonos. La "relación tensionada entre literatura y política" (De Diego 401) que caracterizó las lecturas denuncialistas tuvo algunos de sus desarrollos más renovadores en los debates sobre el realismo que se suscitaron en distintos sectores de la izquierda argentina. Esos debates encontraron un ámbito propicio en los cursos y publicaciones del Instituto de Letras. David Viñas dictó en 1961 - año clave, si se recuerda que es el de la publicación de Realismo y realidad en la narrativa argentina, de Juan Carlos Portantiero- el seminario "Literatura argentina y realidad política" en el que presentó las núcleos centrales del libro que editaría poco después. Al año siguiente, Prieto estuvo a cargo de otro seminario, "Literatura y peronismo", que incluía, entre los textos del programa, Las arenas de Miguel Ángel Speroni, fudas pide una lágrima de Leo Salas y Uno de Elvira Orphée $^{12}$. El artículo de Rosa manifiesta un conocimiento embrionario, todavía intuitivo, de los problemas que se plantean en estas discusiones, intuicionismo que se acentúa si se lo contrasta con la reseña sobre Cabecita negra que Miguel Briante publica en el número 25 de El escarabajo de oro, también en noviembre de 1964 .

El mismo número que se abre con la nota sobre Rozenmacher se cierra con otra colaboración de Rosa, dedicada a Jean Paul Sartre. El motivo, analizar el rechazo de Sartre al Premio Nobel de Literatura ocurrido en octubre de ese mismo año. Entre las razones que lo habrían llevado a rehusar la distinción, Rosa reconoce, contra las difundidas opiniones de Ernesto Sábato (que subrayan la contradicción de que siendo un merecedor incuestionable del premio, resuelva no aceptarlo), los méritos de la capacidad autocrítica de Sartre, su constante disposición a examinar los propios presupuestos. "Vigía permanente de sí mismo", sus decisiones obedecerían a un indeclinable ejercicio de autocuestionamiento. "Tildado de ambiguo, no es sino un hombre responsable de su destino y por tanto del destino del mundo. Acusado de contradictorio, no es sino un hombre solo y lúcido frente al destino del mundo" (nro. 3/4, 45). La imagen de Sartre que Rosa postula, una imagen afín a la de otros defensores contemporáneos, no solo

noviembre de 1958 asumirá Prieto). Entre 1961 y 1963 dictó seminarios en la carrera. Durante 1964 reemplazó a Prieto en "Literatura Argentina I", mientras este se tomó licencia en la materia por sus funciones en el decanato. En 1965 volvió a dictar un seminario. Para una presentación de la intensa y variada actividad que Prieto desplegó en esos ámbitos, ver Podlubne. Toda esta información consta en los registros académico-administrativos de la facultad.

Aunque no fue posible contrastar este dato con los registros institucionales, ya que no se conservan los programas de la época, Norma Desinano, discípula de Prieto en los años del Instituto y actual profesora titular de las cátedras de "Socio"y "Psicolingüística" de la Facultad de Humanidades y Artes de la UNR, asegura haber cursado este seminario y es ella quien recuerda los autores y títulos que se mencionan. 
interesa por el juicio establecido en torno al episodio del Nobel, sino también por la valoración que introduce sobre la trayectoria de Sartre en general. El Sartre que ingresa a Setecientosmonos a través suyo es un intelectual que, plenamente implicado en la acción política directa, se encuentra sin embargo advertido de los riesgos e insuficiencias que encierra la teoría del compromiso. Escribe Rosa: Cuando en su famoso ensayo sobre "Qué es la literatura" esboza su idea de compromiso Sartre se ve abocado a la necesidad de conectar sus teorías con su propia vida. Tenía ahora su propio compromiso. De inmediato se vuelca de lleno a la acción política y funda la Unión Democrática Revolucionaria, que consigue la adhesión de unos pocos intelectuales y la de ningún obrero. El fracaso [concluye] le permite darse cuenta de dos premisas fundamentales sobre las cuales asentar su acción futura: primero, que un verdadero intelectual tiene en sus manos un medio mucho más valioso que la política para hacer efectiva su responsabilidad ante el mundo: su pensamiento, y segundo, la necesidad de someter a revisión ese mismo pensamiento (44).

Conviene recordar que 1964 es un año clave en la trayectoria de Sartre: además de ser el de la renuncia al Nobel, lo que motiva una sorpresa a escala internacional, es el de la publicación de Les mots, su autobiografía, en el mes de abril, y de Situations IV, en junio ${ }^{13}$. La aparición de Les mots provoca en Francia una "únanime oleada de críticas alabando sus cualidades literarias" (Cohen Solal 580). Con un estilo magistral, Sartre sella su relación con la literatura, celebrando la muerte de la concepción que había respetado de niño. La ceremonia es por lo menos ambigua y las numerosas entrevistas que concede a cuantos se lo solicitan complica aún más la tarea de sus exégetas. Entre las entrevistas, la que dispensa a Jacqueline Piatier en Le Monde alcanza notoriedad al reavivar en varios periódicos franceses la polémica sobre el rol del escritor y las funciones de la literatura que lo había tenido en el centro desde la mitad de los años cuarenta ${ }^{14}$. Es probable que Rosa estuviese al tanto de estas discusiones al momento de escribir la nota

13 Sobre los acontecimientos que pautan la importancia de 1964 en la vida de Sartre, ver Cohen Solal 475-691. Para una interpretación de los sucesos que desencadena el rechazo del premio Nobel, ver Kristeva.

14 La entrevista de Piatier se publica en el 18 de abril de 1964. Los escritores Ives Berger y Claude Simon son los primeros en responder a las declaraciones de Sartre. Sus artículos "Nosotros no somos traidores" y " $\mathrm{i} Y$ entonces por qué escribe?", respectivamente, aparecen en $L^{\prime}$ Express, el 28 de mayo. El 10 de junio, Pierre-Henri Simon, el crítico literario de Le Monde, aludido en la polémica, presenta sus posiciones en "Los escritores contra Sartre". Por último, el artículo de Bernard Pingaud, "Pro y contra Sartre", aparece en $L^{\prime}$ Express el 9 de julio. 
para Setecientosmonos. La editorial Losada publicó las traducciones de los dos libros de Sartre ese mismo año y Jorge Álvarez compiló las intervenciones de la polémica en el volumen Los escritores contra Sartre. Pocos meses después de la publicación de la nota de Setecientosmonos, Rosa coordinó en el Instituto de Letras de Rosario dos charlas-debate sobre el libro de Jorge Álvarez ${ }^{15}$.

Aunque es difícil establecer cuánto le debía el artículo de Rosa a la lectura de este debate, o más aún a sus primeros acercamientos al Barthes de Le degré zéro... y de los Essais critiques, lo cierto es que sus conclusiones perciben la complejidad del pensamiento sartreano, antes incluso de poder determinar con nitidez los alcances. La figura del Sartre autoreflexivo que propone Rosa se encuentra más cerca de la intelectual que Barthes presenta en "La literatura hoy", la entrevista publicada en Tel quel en 1961 y luego traducida por el propio Rosa para el número 9 de Setecientosmonos, que de la del hombre de acción, refractario a los problemas estéticos, contra la que disparan sus adversarios en la polémica sobre Le mots. "Uno no se salva más por la política que por la literatura", declara Sartre a Piatier (Los escritores contra Sartre 14) y algo de esta declaración, desoída por sus antagonistas franceses, reverbera en el juicio de Rosa al afirmar que "Es evidente que Sartre ha ido reduciendo modestamente el calibre de sus verdades. Cada vez más pequeñas, pero cada vez más verdaderas" (nro. 3/4, 45). El interés en enfatizar el estado de interrogación permanente que dinamiza las posiciones de Sartre aproxima sus acciones a la idea barthesiana de que la responsabilidad del escritor reside en "comprometer su obra en el mundo, en los problemas del mundo, y, a la vez, suspender ese compromiso precisamente en el momento en que las doctrinas, los partidos, los grupos y las culturas le soplan una respuesta" (nro 9, 2).

Sin advertir las reservas y matices que el Sartre de Rosa introduce sobre el asunto, la "Carta de la dirección" que encabeza el número 5 de Setecientosmonos anuncia el giro comprometido de la revista en la misma lengua intuitiva y estereotipada de las cartas anteriores. Mientras la que abre el número 3/4 presenta a sus integrantes en una actitud autocrítica, conscientes de la necesidad de un cambio editorial e inseguros de los resultados, la del número 5 los muestra demasiado convencidos y ufanos del camino elegido. "El hecho es que en este número decidimos ponernos en contra de medio mundo. Siempre lo estuvimos, hoy nos comprometemos expresamente" (nro. 5, 1). Identificados con un credo que reducen a su expresión mínima ("comprometerse es oponerse"), los directores

15 Debo esta información a Nora Avaro, quien trabaja actualmente con el Archivo personal Adolfo Prieto, en el que se conservan registros de esta y otras actividades del Instituto. 
distinguen una larga serie de adversarios, de estatuto diverso, cifra clara de la ansiedad con que asumen este posicionamiento:

[...] estamos en contra de toda literatura oficial; de los suplementos literarios de casi todos los diarios (entiéndase: La Capital, La Prensa, La Nación, etc.); del paternalismo oficioso; de la literatura gratuita, de los clanes académicos (SADE); de la pedantería universitaria; de los que creen que la literatura argentina comienza con Borges y termina con Sábato; de toda forma de alienación moral, intelectual, económica (que no es sino una, ya provenga de la Curia Vaticana, del Kremlin o del Pentágono).(nro. 5, 1)

El imperativo del compromiso político se anuncia con la misma falta de reflexión y rudimentos de estilo que identifica a las editoriales anteriores. Lo nuevo es la indignación: los monos ya no están contentos como anunciaba, pocos meses antes, el número doble, sino que se muestran indignados y de esa indignación procede, y a ella se limita, la necesidad de pronunciarse:

No pretendemos aportar datos o elementos deslumbrantes o sensacionales. No pretendemos constituirnos en los dueños de la verdad ni en la fuente obligada para la interpretación de la realidad. No pretendemos que nuestros testimonios resulten estupendos análisis legales, estadísticos, políticos o sociales de los hechos que nos ocupan. Queremos sí, manifestarnos, dejar constancia, tomar partido..., queremos en pocas palabras, hablar claro, indicar lo detestable y lo intolerable de actitudes determinadas... (Testimonios, 1)

El compromiso tiene propósitos declarativos, testimoniales, ni siquiera denuncialistas. El editorial con que se abre el suplemento Testimonios parece incluso eludir el verbo "denunciar" (recurre a "señalar", "indicar", pero nunca a "denunciar"); se trata básicamente de "hacer saber", de dar a conocer lo que los medios de difusión, el periodismo oficial, escatiman o tergiversan. Si bien es cierto que varios de los miembros de la revista asumen luego una militancia directa en distintos sectores de la izquierda y participan de acciones políticas concretas, resultaría excesivo atribuir a la intervención de Setecientosmonos un alcance ideológico de consecuencias relevantes $^{16}$. Ni el número 6, aparecido en agosto de 1966, es decir a las pocas semanas, ni los números posteriores, se pronuncian contra el golpe de Onganía.

16 Sobre la participación política de algunos de los integrantes, ver Garbatzky. Garbatzky encuentra en la revista sucesivas articulaciones entre vanguardia literaria, vanguardia artística y vanguardia política. Estas articulaciones prepararían el camino a la experiencia estéticopolítica que culminó en Tucumán arde. 
Varias décadas después, durante la mesa redonda sobre el rol de las revistas culturales que mencioné en el inicio, Rosa describe los efectos de esa intervención en términos acotados y atribuye las reyertas ideológicas menos a las posiciones políticas de los protagonistas que a las lecturas teóricas que comienzan a difundirse en la publicación:

[...] la revista nos produjo una serie de pequeñas cositas como, por ejemplo, enojarnos simultáneamente con distintas instituciones tradicionales [...] Salió de ahí una especie de panfleto donde yo era, de alguna medida, una suerte de anticristo por las cosas que decía, aunque en última instancia, proponía la lectura del Saint Genet de Sartre que para la Iglesia provinciana podía ser bastante cuestionable. ("El rol de las revistas culturales" viii)

Sin desconocer la voluntad autofigural que alienta la elección de la imagen del anticristo para describir sus comienzos, el recuerdo de Rosa coincide en parte con el de Martini cuando cuenta que sus rivales de El arremangado brazo los acusaban de "afrancesados", de "propiciar una literatura más de élite". En el imaginario local, el denuncialismo era la marca de la revista que Rafael Ielpi, Romeo Medina y Aldo Oliva dirigieron entre 1963 y $1964^{17}$. La rivalidad entre ambas publicaciones alimenta todavía el folklore de la época. Si se atiende a las referencias teóricas a las que recurren unos y otros, la distancia se torna evidente: los miembros de El arremangado... se mantienen apegados a las tesis de Lucien Goldmann ${ }^{18}$.

\section{Modernización teórica y nueva crítica}

Con el número 6 (agosto 1965), Setecientosmonos se transforma de modo sustancial. Nicolás Rosa se incorpora a la dirección, la revista cambia de formato: estrena el elegante diseño Mondrian, a cargo de Rodolfo Elizalde, y las cartas-editoriales,

17 El primer número se publicó en septiembre de 1963 y el segundo en agosto de 1964. Los dos primeros números de Setecientosmonos aparecieron en mayo y julio de 1964, respectivamente. Esto es, entre los números de El arremangado...

18 Los números de El arremangado... comienzan con las traducciones de la primera y segunda parte de "Materialismo dialéctico e historia de la literatura", de Goldmann, a cargo de Noemí Ulla y Romeo Medina. El artículo, publicado inicialmente en la Revue de metaphysique et de morale 3 (París, 1950), se incluye luego en Recherches dialectiques (Paris: Gallimard, 1959). Para una presentación general de esta revista, ver Gerbaudo. Sobre el vínculo entre ambas publicaciones, es interesante consultar la extensa reseña al libro de poemas El vicio absoluto (Rosario: Vigil, 1966), de Rafael Ielpi, que Luis María Castellanos escribe en el número 9 de Setecientosmonos. Aguirre ("En el reino de la literatura" 21) encuentra en esta reseña una suerte de reconciliación entre ambas publicaciones. 
que hasta entonces habían aparecido en las primeras páginas, se sustituyen por textos de teóricos franceses contemporáneos, traducidos por el propio Rosa: un capítulo de Sentido y sin sentido de Maurice Merleau-Ponty, un fragmento del Saint Genet de Sartre, otro de Mitologías de Roland Barthes y la entrevista a Barthes "La literatura hoy", aparecida inicialmente en Tel Quel, en 1961, e incluida luego en Essais critiques (París: Éditions du Seuil, 1964) ${ }^{19}$. El cambio imprime a la revista un acento nuevo que restringe, casi por completo, el impresionismo crítico y el amateurismo literario de los comienzos. Setecientosmonos se compone en adelante al ritmo acelerado de la actualización teórica de Rosa y de su particular percepción de las transformaciones que afectan el discurso de la crítica argentina en ese momento. "Literatura argentina y David Viñas" y "Sexo, traición, Masotta y Roberto Arlt", las notas bibliográficas publicadas en los números 5 y 6 respectivamente, resultan un registro inestimable de esa percepción.

La importancia de la nota sobre Viñas deriva no solo del valor documental que le confiere ser la primera lectura de Literatura argentina y realidad política, el libro que veinte años después se convierte en un clásico de la crítica literaria y cultural argentina, sino también de la perspicacia que acredita para entonces el pensamiento crítico de Rosa ${ }^{20}$. El comentario avanza entre el reconocimiento y la evaluación exigente. Junto a "Portantiero, Sebreli, Prieto, Jitrik, Mazotta [sic]", escribe Rosa, Viñas representa "esos nuevos intentos críticos que posibilitan la valoración real y total de la obra literaria en una dualidad inseparable: su contexto social y su individualidad estética"(nro. 5,13$)$. El interés por la obra de Viñas recorre de aquí en adelante los ensayos de Rosa. El requerimiento escrupuloso y la observación atinada deciden un modo de lectura sopesado que se perfilará con los años. La nota de Setecientosmonos enuncia elogios y objeciones que los lectores desarrollarán tiempo después. El joven Rosa comparte la matriz sociológica, fuertemente historicista, que orienta el "movimiento de renovación" de la crítica argentina y celebra la tarea "desmitificadora" que realiza Literatura argentina y realidad política. El acuerdo no esquiva sin embargo las debilidades metodológicas y los fallos argumentativos del volumen. Con impulso certero y ruidoso, propio de quien se está haciendo algo tardíamente un lugar en la escena de la renovación crítica (debía tener alrededor de 30 años en ese momento), Rosa discute determinismos, reducciones, antinomias, excesos y vaguedades interpretativas de Viñas.

19 "Novela y metafísica" de Merleau-Ponty apareció en el nro. 6, "Santidad y consumo" de Sartre, en el nro. 5 y "Los mitos de la burguesía" de Barthes, en el nro. 8.

20 Agradezco a Analía Capdevila la colaboración en el trabajo de archivo para confirmar este dato. 
Entre las objeciones que presenta, hay una en particular que me interesa mencionar: la que apunta a la célebre cláusula inaugural del libro. Rosa cuestiona el matiz esencialista que se filtra en el comienzo de Literatura argentina y realidad política, al encontrar desafortunada la identificación del comienzo de la historia literaria argentina con el nacimiento y evolución de la "voluntad nacional". Se recordará que la primera edición empieza afirmando, en alusión directa a su origen romántico, "La literatura argentina es la historia de la voluntad nacional".

La expresión 'voluntad nacional' [sostiene Rosa] está tan cerca del "espíritu del pueblo" o "espíritu de la época" del romanticismo herderiano y de los irracionalismos nacionalistas barresianos, que produce desconfianza. La expresión es hueca. Le falta realidad.

$[\ldots]$

[Viñas] debió, tal vez, encabezar el análisis de "La mirada a Europa" con una clara introducción de lo que entiende por 'voluntad nacional' pues corre el riesgo de caer en el mismo error que critica: un término vago, impreciso y abstracto señalado como motor invisible del proceso histórico. (nro. 5, 9-10)

Sin renunciar a la cláusula observada, Viñas modifica ese comienzo en la siguiente edición. La versión parcial que en 1971 reedita Jorge Álvarez con el subtítulo De Sarmiento a Cortázar se inicia con la conocida metáfora de la violación y reserva un sitio menos visible para la idea de voluntad nacional, que pasa a encabezar el primer capítulo de la segunda parte, "Rosas, romanticismo y literatura nacional" ${ }^{\prime 21}$. Aun cuando en ediciones posteriores combine ambas, la imagen de la violación sustituirá a la de la voluntad nacional en la memoria de los lectores. Me pregunto cuánto habrá incidido en este reemplazo el comentario de Rosa, que sin dudas Viñas conocía. ¿Podría pensarse el relevo como una respuesta a sus observaciones, como el principio de una larga conversación compartida? ¿Habría conseguido el joven Rosa el reconocimiento del para entonces acreditado novelista y crítico prominente?22 Alejandra Laera ("Para una historia de la literatura argentina") leyó las consecuencias derivadas de esta y otras sustituciones

21 Cuando se publica esta edición, Rosa vuelve a escribir una extensa nota, titulada "Viñas: la evolución de una crítica (Literatura y política)", en la revista Los libros nro. 18 (1971). A diferencia de la de Setecientosmonos, que no recoge en volumen, "Viñas: la evolución de una crítica (Literatura y política)" se incluye en Los fulgores del simulacro (Santa Fe: Universidad del Litoral, 1987), con el título retocado: "Viñas: las transformaciones de una crítica".

22 En 1965, Viñas había publicado varias de sus novelas más importantes: Cayó sobre su rostro (1955), Los años despiadados (1956), Un dios cotidiano (1957), Los dueños de la tierra (1958) y Dar la cara (1962). 
y desplazamientos que afectaron las sucesivas reescrituras del comienzo de Literatura argentina y realidad política. Sus conclusiones demostraron que, incluso apelando a la metáfora de la violación, Viñas no logró sustraerse nunca a una "suerte de esencialismo idiosincrático que parecía superado largamente por una perspectiva dialéctica [...] El esencialismo [sostiene Laera] acecha en el 'revés de trama' del denuncialismo que practica Viñas (166). La observación del joven Rosa identificaba un rasgo que Laera precisa idiosincrático.

Como la nota sobre Viñas, "Sexo, traición, Masotta y Roberto Arlt" resulta también la primera reseña a otro clásico de la crítica literaria argentina ${ }^{23}$. Es probable que Rosa conociera con anticipación los artículos que integran el volumen: "Silencio y comunidad" había aparecido, con el título "Silencio y humillación en Roberto Arlt", en El Litoral de Rosario, el 6 de agosto de 1958; "La plancha de metal", en el nro. 13 de la revista Centro, en 1959, y "Seis intentos frustrados de escribir sobre Arlt", en Hoy en la cultura, nro. 5, en 1962. A quien probablemente Rosa no conocía todavía era al propio Masotta. Ambos se encontrarían unos años después cuando Crítica y significación, el primer libro de Rosa, se presentase en Buenos Aires ${ }^{24}$.

¿Habrá sido Masotta el presentador o el detractor de Crítica y significación? ¿Sabría de la nota de Rosa sobre Sexo y traición...? Queda para otra oportunidad el comentario de algunos sucesos intermedios que podrían acercar una respuesta conjetural. No obstante, más allá de las respuestas a estos interrogantes, la nota de Setecientosmonos registra el cruce inicial de dos trayectorias críticas con evidentes convergencias posteriores. Mientras Viñas será un objeto de atracción persistente a lo largo de la obra de Rosa, Masotta le indicará (o le confirmará, según se estime) el camino a seguir: el de la exigencia metodológica derivada de la modernización teórica. El elogio del método, la precisión con que Masotta dispone del "instrumento crítico" que le provee la fenomenología existencial y los resultados que obtiene de la lectura de Arlt, definen las líneas centrales de la nota: Masotta [concluye Rosa] hace un valioso uso de este instrumento, llegando por momentos a la clarividencia, justo allí, cuando la develación del sentido posible de una relación coincide con la expresión exacta, el verbo justo. Por

23 El agradecimiento es para Diego Peller que certificó el dato con sus investigaciones.

24 Existen diferentes versiones de este encuentro. Rosa cuenta que Masotta tuvo a su cargo la presentación del libro en una reunión privada en casa de Ricardo Piglia ("Entrevista a Nicolás Rosa" por Rodrigues de Andrade). Piglia recuerda, en cambio, que Masotta asiste a esa reunión, convocada por los miembros de la revista Los libros (Héctor Schmucler, Josefina Ludmer, Germán García, entre otros) y acusa a Rosa de haberle "copiado" todo. "Entonces se arma un debate increíble, donde Nicolás queda completamente acorralado" (Somoza y Vinelli 14). 
momentos, el estilo de Masotta se vuelve oscuro, confuso: la materia es difícil, se allega por aproximación, por tanteo dialéctico, si es permisible llamarlo así. El rigor de su método clarifica simultáneamente al lector y al propio autor. Hay unidad entre el planteo y la demostración, entre lo que se propone y lo que se consigue. Claro es que quedan fuera de su ámbito numerosos problemas que la novelística de Arlt presentaba. No es un error: es precisión metodológica. (nro. 6, 28)

Algunos párrafos antes, Rosa celebraba que Masotta hubiese caracterizado la obra de Arlt a partir de dos temas fundamentales: la traición y el sexo; ese recorte le habría permitido dar con la "unidad sintética de sentido" ajustada a esta literatura. A esa decisión, que juzgaba entre los principales aciertos del crítico, le objetaba sin embargo una debilidad. Una vez más el reconocimiento reservaba su lugar a la réplica. Masotta, indicaba Rosa, no atendía a la relación dialéctica subyacente entre ambos temas y simplificaba ese vínculo al incurrir en un determinismo impropio de alguien con sus alertas analíticas. El comentario aplicaba a la lectura de Masotta el rigor que había aprendido en sus ensayos y se acercaba sin saberlo a las reservas sobre el libro que el autor había expuesto pocos meses antes en "Roberto Arlt, yo mismo" .

El acercamiento no obedecía solo a la convergencia de objetos e intereses. Un itinerario de lecturas comunes marcó, en el horizonte amplio de una reinterpretación de la filosofía marxista, el tránsito raudo de toda una generación desde la fenomenología existencialista de Sartre y Merleau-Ponty hasta lo que sin demasiadas precisiones se designa como el estructuralismo de Levi-Strauss, Barthes, Lacan. "Masotta [dijo Beatriz Sarlo (421)] era entonces una especie de modelo de intelectual moderno". Rosa se convertiría en otro. Vicenç Tuset ("La crítica literaria frente al estructuralismo") sintetizó diferencias importantes en los proyectos intelectuales de ambos. Lo cierto es que la serie de textos teóricos que Rosa tradujo y publicó en Setecientosmonos son índice de la premura y la avidez con que procesa ese tránsito. Rosa cuenta que Masotta le dijo por entonces: "Nicolás, cuando se traduzcan los textos franceses que leíamos para estar más

25 Escrito a pedido de Jorge Álvarez para la presentación de Sexo y traición..., "Roberto Arlt, yo mismo" se inicia con la crítica de Masotta a los recursos con los que contaba en ese momento. "Pero volvería yo a escribir este libro, ahora, si no estuviera ya escrito? Bien, creo que no podría hacerlo. Entre otras cosas, porque hoy soy menos ignorante que entonces, más cauteloso. Y seguramente: una cierta indigencia cultural, de formación, con respecto a los instrumentos intelectuales que realmente manejaba, estoy seguro, fueron entonces el motor que no sólo me impulsó a planear el libro, sino que me permitió escribirlo" (87). 
a la moda nos van a llamar traductores". En su recuerdo, la escena alcanza vigor romántico. "Me sentí molesto, agrega Rosa, pero con el pasar del tiempo la herida narcisista se fue curando cuando leí a Echeverría y a Sarmiento, también ellos verdaderos "traductores' de la cultura francesa" (Rosa, "Entrevista" 137).

Imbuidas de un retrospectivo hálito fundacional, las traducciones de Setecientosmonos responden a las necesidades y la ansiedad que definen los aprendizajes de Rosa, antes que a decisiones editoriales o perspectivas compartidas por sus miembros. La revista funciona como el ámbito de presentación, elaboración y difusión de esos nuevos aprendizajes. En sus páginas, Rosa introduce, glosa, parafrasea, discute y usa esos saberes diversos y todavía inestables. Setecientosmonos cuenta la novela de formación del crítico: las primeras elecciones teóricas, los vínculos e influencias iniciales y sus tentativas heurísticas. "Escribimos una revista [dice Rosa ("El rol de las revistas culturales" viii)] porque todavía no podemos escribir un libro, porque todavía no podemos armarlo". El enunciado soslaya el espíritu gregario que suele alentar estas publicaciones para afirmar en su lugar una verdad autobiográfica. Los artículos dedicados a Sartre, Julio Mafud y Guillermo Cabrera Infante, publicados respectivamente en los números 7 (diciembre 1965), 9 (junio 1967) y 10 (octubre 1967) de Setecientosmonos, componen, casi en su totalidad, Crítica y significación, editado en Galerna en $1970^{26}$. El primero de estos artículos acompaña la traducción del Saint Genet y presenta una glosa profusa del pensamiento del autor en ese libro. El dedicado a Mafud, un texto de coyuntura, combate las banalidades de La revolución sexual argentina, éxito de ventas del año anterior, con un arsenal teórico desproporcionado: Marx, Barthes y Lacan, entre otros. "Tres tristes tigres: una patología del lenguaje", el más consistente de los tres, el único que conservaría alguna eficacia actual, conjuga el análisis de la lengua poética en Cabrera Infante, a partir de una matriz estilísticojakobsoniana, con las perspectivas contemporáneas sobre el género novela. El análisis parte de una crítica severa al valor comunicativo de la prosa suscripto por Sartre $^{27}$. Los artículos duplican en cierta forma el itinerario teórico presentado en las traducciones. La reseña sobre Crítica y significación que Josefina Ludmer publica en la revista Los libros en 1970 subraya entre los desaciertos del volumen la dificultad para sintetizar la diversidad de metodologías a las que apela ${ }^{28}$.

26 Para una presentación comentada de las colaboraciones de Rosa en la revista, ver Di Crosta.

27 Afirma Rosa: "Ver como excluyente el carácter comunicativo del lenguaje sería negar su sentido de creación, su función estética, y ha llevado a gruesos errores: por ejemplo la bastarda distinción que establece Sartre entre prosa y poesía en Situations II" (nro. 10,3).

"La literatura abierta al rigor", Los libros nro. 9 (1970): 5. 
Paralelo al capítulo dedicado a la formación teórica de Rosa, Setecientosmonos cuenta también el de sus primeras relaciones intelectuales. La crítica universitaria ingresa a la revista por iniciativa suya. Rosa invita a Adolfo Prieto y a los miembros de su equipo y consigue que varios - Prieto mismo, entre ellos- envíen sus colaboraciones. En el equipo se encuentran Josefina Ludmer, María Teresa Gramuglio y Norma Desinano, que acaban de terminar la carrera de grado, y Gladys Onega, cuyo primer libro, La inmigración en la literatura argentina, aparecido unos meses antes en la colección de Cuadernos del Instituto de Letras (mayo 1965), había sido elogiosamente reseñado por Rosa en el número 6 de la revista. Las colaboraciones de los críticos universitarios se publican en los últimos cuatro números e introducen el interés por la nueva narrativa ligada al llamado boom de la literatura latinoamericana. En conjunto, brindan un panorama de los asuntos y autores que se discuten en las aulas del Instituto de Letras, antes y durante los meses previos a la intervención de Onganía. Los artículos de Prieto y Onega componen, junto con el de Rosa Boldori, que procede también de la universidad pero no participa del equipo de Prieto, el dossier "Cortázar o el fin del juego", que se publica en el número $7^{29}$. Pocos meses después, el volumen 6 (agosto 1966) del Boletín de Literaturas Hispánicas de la Universidad Nacional del Litoral, el último que sale bajo la dirección de Prieto, recopila textos de los mismos (y otros) autores en una edición también dedicada a Cortázar. Será preciso leer estos artículos en el marco amplio de la recepción crítica que recibe la obra de Cortázar en Argentina para evaluar sus alcances ${ }^{30}$.

Las colaboraciones de Ludmer y Gramuglio (la primera, en su caso, porque seguirá publicando en entregas posteriores) aparecen en el número 8 (agosto 1966) y analizan las novelas Estudio Q, de Vicente Leñero, y Pedro Páramo, de Juan Rulfo, respectivamente ${ }^{31}$. Ambos textos, registro de las iniciaciones críticas de las autoras, son resultado directo de las clases que, a propuesta de Prieto, Ludmer, Gramuglio y Desinano dictan en el seminario "Realismo en la novela hispanoamericana contemporánea", entre mayo y septiembre de $1965^{32}$. En el

29 Me refiero a "Julio Cortázar, hoy", de Adolfo Prieto, "Cortázar: personaje y misterio" de Gladys Onega y "Cortázar: una novelística nueva", de Rosa Boldori.

Esta evaluación deberá retomar las conclusiones que Graciela Montaldo ("Destinos y recepción") propone en su estudio sobre la recepción de Rayuela e incorporar los artículos de Setecientosmonos y el Boletín de Literaturas Hispánicas al recorrido de lecturas críticas que analiza.

31 Sus títulos son "Estudio Q: una novela sobre la novela" y "Juan Rulfo: Pedro Páramo". En el número siguiente, Gramuglio publica "La actitud testimonial de David Viñas" y una reseña a Los nuestros, el libro de Luis Harss. En el número 10, aparece su estudio sobre la novela objetivista. 
mismo número en el que aparecen los artículos de Ludmer y Gramuglio, Desinano publica una reseña a Todos los fuegos el fuego, de Cortázar, pero su colaboración más significativa es sin dudas la que dedica, en el número siguiente, a la narrativa de Juan José Saer, un texto precursor de la extensa recepción que el escritor tuvo a partir de ese momento ${ }^{33}$. Saer colabora en el último número de Setecientosmonos con la traducción del cuento "La playa" de Alain Robbe Grillet y una selección de poemas que más adelante incluye en El arte de narrar (Caracas: Fundarte, 1977). En la nota de presentación a los poemas, se lee: "Juan José Saer, uno de los más destacados novelistas actuales que apuntan experimentalmente a una renovación de la narrativa argentina, es también poeta" (nro.10, 21). Juan L. Ortiz y Hugo Padeletti, poetas próximos a Saer y a los críticos universitarios, habían colaborado antes. En el número 6, se incluyeron los poemas "La muchachita", que Ortiz incorporó luego a La orilla que se abisma (Rosario: Editorial Vigil, 1970) y "Lo auténtico", que Padeletti publica años después en Poemas 1960/1980 (Santa Fe: Universidad Nacional del Litoral, 1989), con una dedicatoria a Rosa.

El interés por las nuevas formas y posibilidades del realismo en la literatura hispanoamericana es el eje que nuclea las lecturas de los críticos universitarios. La reflexión sobre las distintas variantes que en este contexto ofrece el género novela encuentra en las referencias al noveau roman, tendencia a la que Gramuglio dedica su estudio "El espacio en la novela objetivista", un ámbito compartido al que estos textos apelan tanto para analizar el funcionamiento de las nuevas técnicas narrativas como para evaluar las relaciones entre la literatura y el mundo.

y editado por Paidós en 1974. El volumen alcanza una doble relevancia programática, en la medida en que se presenta como "la nueva narrativa por la nueva crítica", para citar los términos de Lafforgue. Antes de este artículo, Ludmer había publicado "Ernesto Sábato y un testimonio del fracaso" en el nro. 3 del Boletín de Literaturas Hispánicas del Instituto de Letras.

33 Con el título "J. J. Saer: después de la vuelta completa", el artículo de Desinano recoge las discusiones que en torno a la obra del escritor se venían suscitado en el Instituto de Letras desde 1960. Cuando se edita En la zona, el primer libro de cuentos del escritor, Prieto organiza en el Instituto de Letras una mesa redonda sobre el libro. Los testimonios coinciden en señalar que, además de Saer, Prieto y Gramuglio, asisten al debate Aldo Oliva, Norma Desinano y Noemí Ulla. Es muy probable que el poeta Hugo Gola, amigo personal del escritor, también estuviera presente, aunque no fue posible corroborar este dato con exactitud. Valorada en forma retrospectiva, esta mesa redonda da el impulso inicial a la larga historia de la recepción de Saer en Argentina. Cuenta Nora Catelli (217) que, en 1965, Gladys Onega incluye Responso, editada el año anterior, entre las lecturas obligatorias de un seminario de crítica que dicta en el Instituto. Al año siguiente del artículo de Desinano, Prieto menciona a Saer en su libro Literatura y subdesarrollo y lo incluye en el Diccionario básico de la literatura argentina, publicado en la colección Capítulo de CEAL. En 1969, Gramuglio publica "Las aventuras del orden" en Los libros. Para una reconstrucción crítica documentada de las lecturas sobre Saer, es imprescindible ver Dalmaroni. 
El artículo de Gramuglio procede de los apuntes de una conferencia que dicta por entonces en el Centro de Estudios de Filosofía, Letras y Ciencias del Hombre $^{34}$. Las conversaciones con Saer, que en julio de 1968 viaja a París con una beca para estudiar ese movimiento y entrevista a las figuras más representativas, deben haber incidido en su visión del tema. Seguramente Rosa también participaría de estas charlas. En 1966 había publicado el artículo "Michel Butor y la nueva realidad" en el suplemento literario del diario Crónica de Rosario.

Dejo para una próxima oportunidad el comentario de los ecos, los intercambios y las réplicas del intenso diálogo que resuena en estas colaboraciones de Setecientosmonos. No obstante, a modo de conclusión parcial y provisoria, diría que, en el mismo sentido en el que Prieto (23) anotó que los primeros artículos de Rosa ilustran el pasaje del existencialismo sartreano a la lectura barthesiana del texto literario como reflexión sobre la lengua que lo actualiza, los que él y su equipo publican en la revista describen los esfuerzos de la sociología literaria por distanciarse de la sociología empírica y articularse con las demandas de método que exigen las nuevas técnicas analíticas del estructuralismo. Con la inclusión de los críticos universitarios, Setecientosmonos cuenta, junto al relato de formación de Rosa, un episodio central de lo que el mismo Rosa designó como "la novela familiar de la crítica argentina": el que reúne a varios de sus representantes más destacados en una conversación inaugural, a la que accedemos, de modo fragmentario, interpelando las páginas de la revista.

\section{Obras citadas}

Aguirre, Osvaldo. "En el reino de la literatura". Setecientosmonos: Antología. Eds.

Osvaldo Aguirre y Gilda Di Crosta. Buenos Aires: Santiago Arcos, 2012. 11-25. Impreso.

Álvarez, Jorge. Mis memorias. Buenos Aires: Libros del Zorzal, 2013. Impreso.

Benjamin, Walter. La dialéctica en suspenso: fragmentos sobre la historia. Traducción, introducción y notas de Pablo Oyarzún Robles. Santiago de Chile: LOM Ediciones, 1995. Impreso.

34 El Centro de Estudios de Filosofía, Letras y Ciencias del Hombre (CEF) fue fundado en 1966 por los profesores renunciantes de la facultad, con el propósito de mantener activa la tarea docente y de investigación desarrollada en las aulas universitarias. Su diseño institucional aspiraba inicialmente a reproducir el de los institutos de la facultad: Prieto fue su director durante el primer año. El centro funcionó como una suerte de instituto independiente de estudios superiores y ofreció cursos, charlas y conferencia sobre temas de distinta índole, para un público general y unos pocos estudiantes universitarios que siguieron vinculados a los profesores. 
Calabrese, Elisa. "Animales fabulosos: un proyecto cultural comprometido". Animales fabulosos: las revistas de Abelardo Castillo. Eds. Elisa Calabrese y Aymará de Llano. Mar del Plata: Editorial Martin, 2006. 11-29. Impreso.

Catelli, Nora. "Los desplazamientos innecesarios: prólogo a El concepto de ficción". Zona de prólogos. Comp. Paulo Ricci. Buenos Aires: Seix Barral, 2011. 217-229. Impreso.

Cohen Solal, Annie. Sartre: una vida. Trads. Agustín López Tobajas y Christine Monot. Buenos Aires: Emecé Ediciones, 1990. Impreso.

Dalmaroni, Miguel. "El largo camino del 'silencio' al 'consenso': la recepción de Saer en Argentina (1964-1987)". Glosa y El entenado. Ed.Julio Premat. Córdoba: Alción, 2010. 607-663. Impreso.

Di Crosta, Gilda. "Nicolás Rosa y la nueva crítica literaria". Setecientosmonos: Antología. Eds. Osvaldo Aguirre y Gilda Di Crosta. Buenos Aires: Santiago Arcos, 2012. 109118. Impreso.

De Diego, José Luis. "Los intelectuales y la izquierda en la Argentina (1955-1975)". Historia de los intelectuales en América Latina. Dir. Carlos Altamirano. Buenos Aires: Katz, 2010. 393-416. Impreso.

Derrida, Jacques. Mal de archivo: una impresión freudiana. Trad. Paco Vidarte. Madrid: Trotta, 1997. Impreso.

De Torre, Guillermo. Problemática de la literatura. Buenos Aires: Losada, 1951. Impreso.

Garbatzky, Irina. "Sentidos de vanguardia en Setecientosmonos: ruptura y eficacia (artística, política, crítica)". Setecientosmonos: Antología. Eds. Osvaldo Aguirre y Gilda Di Crosta. Buenos Aires: Santiago Arcos, 2012. 119-131. Impreso.

Gerbaudo, Analía. "Papeles de la siesta provinciana". Discurso y crítica literaria. Ed. María Angélica Hechim. Centro de Publicaciones de la UNL, 2009. 77-111. Impreso.

Kristeva, Julia. "Sartre, o hay razones para la revuelta". Sentido y sentido de la revuelta: literatura y pisicoanálisis. Trad. Irene Agoff. Buenos Aires: Eudeba, 1998. 251-306. Impreso.

Laera, Alejandra. "Para una historia de la literatura argentina: orígenes, repeticiones, revanchas". Dossier: Literatura argentina y realidad política, un clásico. Prismas: Revista de historia intelectual 14 (2010): 163-167. Impreso.

Los escritores contra Sartre. Buenos Aires: Jorge Álvarez, 1964. Impreso.

Ludmer, Josefina. "La literatura abierta al rigor". Los libros 9 (1970): 5. Impreso.

Martini, Juan. "Liminar". Setecientosmonos: Antología. Eds. Osvaldo Aguirre y Gilda Di Crosta. Buenos Aires: Santiago Arcos, 2012. 7-9. Impreso.

Masotta, Oscar. Sexo y traición en Roberto Arlt. Buenos Aires: CEAL, 1982. Impreso. 
Montaldo, Graciela. "Destinos y recepción”. Cortázar, Julio. Rayuela. Eds. y coords. Julio Ortega y Saúl Yurkievich. Buenos Aires: Colección Archivos, 1991. 597-612. Impreso.

Olguín, Sergio, y Claudio Zeiger. "La narrativa como programa: el realismo frente al espejo". La irrupción de la crítica. Dir. Susana Cella. Buenos Aires: Emecé, 1999. 377-402. Impreso.

Pérez Cantón, Omar. Entrevista. Setecientosmonos: Antología. Eds. Osvaldo Aguirre y Gilda Di Crosta. Buenos Aires: Santiago Arcos, 2012. 344-347. Impreso.

Podlubne, Judith. "Los años Prieto". "La lectora moderna. Apuntes para una biografía intelectual". Gramuglio, María Teresa. Nacionalismo y cosmopolitismo en la literatura argentina. Rosario: Editorial Municipal, 2013. 12-19. Impreso.

Prieto, Adolfo. "Estructuralismo y después". Punto de vista 34 (1989): 22-25. Impreso. Radeff, Rubén. "Entrevista a Rubén Radeff". Por Julieta Tonello. Constelación Santa $\mathrm{Fe} .27$ dic 2012. Web. 12 dic 2014.

Rosa, Nicolás. El arte del olvido. Buenos Aires: Puntosur, 1990. Impreso. "Entrevista a Nicolás Rosa". Por Rosangela Rodrigues de Andrade. Puzzle(s) Masotta. Oscar Masotta: lo imaginario (búsqueda teórica y búsqueda de imágenes matrices). Rosario: Homo Sapiens Ediciones, 1997. 137-141. Impreso.

Sarlo, Beatriz. Entrevista. King, John. El Di Tella y el desarrollo cultural argentino en la década del sesenta. Buenos Aires: Asuntoimpreso Ediciones, 2007. 419-425. Impreso.

Schork, Carlos. Entrevista. Por Julieta Tonello y Osvaldo Aguirre. Setecientosmonos: Antología. Eds. Osvaldo Aguirre y Gilda Di Crosta. Buenos Aires: Santiago Arcos, 2012. 337-343. Impreso.

Setecientosmonos. Colección completa. Rosario: 1964-1967.

Somoza, Patricia, y Elena Vinelli: "Para una historia de Los libros". Revista Los libros. Edición fascimilar. Tomo I (1969-1970). Buenos Aires: Ediciones Biblioteca Nacional, 2011. 9-19. Impreso.

Tuset, Vicenç. "La crítica literaria frente al estructuralismo: ecos locales de un debate internacional". VII fornadas de Sociología de la Universidad Nacional de La Plata. "Argentina en el escenario latinoamericano actual: debates desde las ciencias sociales". 5 al 7 de diciembre de 2012, La Plata. Web. 28 nov 2014.

VVAA. "El rol de las revistas culturales". Espacios 12 (1993): i-xvii. Impreso. 